\title{
Risk Assessment of Lingual Plate Perforation in Posterior Mandibular Region: A Virtual Implant Placement Study Using Cone-Beam Computed Tomography
}

\author{
Hsun-Liang Chan, * Erika Benavides, ${ }^{\dagger}$ Chu-Yuan Yeh, ${ }^{*}$ Jia-Hui Fu, * Ivan E. Rudek, * \\ and Hom-Lay Wang ${ }^{\dagger}$
}

Background: Lingual plate perforation at the time of implant placement in posterior mandible is a potential surgical complication, and presence of a lingual concavity is considered a risk factor. Little is known about the spatial relationship between implant and lingual plate. The role of lingual concavity in the risk of lingual perforation has not yet been fully studied. This computer-simulated study investigates the incidence of lingual plate perforation in edentulous mandibular first molar region and the risk of perforation in the area of lingual concavity.

Methods: One hundred and three qualified cone-beam computed tomography scans were selected from the database. Implants of various dimensions were virtually placed into the area using computer software. The distance between implant tip and lingual plate was measured using a digital caliper. Incidence of lingual plate perforation and proximity of the implant tip to lingual plate were measured for three types of cross-sectional mandibular morphology.

Results: One hundred and three cone-beam computed tomography scans with 118 sites were available for analysis. The intraexaminer and interexaminer agreements were 0.93 and 0.89 , respectively. The predicted incidence of lingual plate perforation was $1.1 \%$ to $1.2 \%$. Most implants, which were within $1 \mathrm{~mm}$ from lingual plate, occurred in sites with lingual concavity (type- $U$ ridge).

Conclusions: This study demonstrates a novel experimental design by which the spatial relation between implant and lingual plate in mandibular first molar region is investigated. Incidence of lingual plate perforation during implant placement is predicted to be $1.1 \%$ to $1.2 \%$ and it will most likely happen in type-U ridge. J Periodontol 2011;82:129-135.

\section{KEY WORDS}

Anatomy; cone-beam computed tomography; dental implants; intraoperative complications; jaw, edentulous; mandible.

\footnotetext{
* Graduate Periodontics, School of Dentistry, University of Michigan, Ann Arbor, MI.

$\dagger$ Department of Periodontics and Oral Medicine, School of Dentistry, University of Michigan.
}

$\tau$ mplant therapy has become an integral part of daily periodontal practice because of its high success rates. Its applications have expanded from long-span edentulous ridges ${ }^{1}$ to single tooth replacement. ${ }^{2}$ The therapeutic goals have migrated from purely functional to restoring lost esthetics and ensuring long-term stability. To meet these objectives, the concept of a prosthetically driven implant treatment plan has emerged. ${ }^{3}$ As a result, implant treatment planning takes on a "crown-down" approach, which means that the treatment plan starts with determining the final location of the restoration before deciding on the implant position.

Implant angulation generally follows the long axis of occlusal forces in the posterior region. ${ }^{4}$ This is because bone can resist compression forces better than tensile or shear stresses; ${ }^{5}$ an axially loaded implant can direct more compressive than tensile or shear forces on bone. In addition, better stress/strain distribution is possible when implants are placed along the axis of loading with multiple areas of cortical contact. ${ }^{6}$ From a practical standpoint, prefabricated abutments are less expensive than customized ones, which are usually necessary with off-angled implant placement.

doi: 10.1902/jop.2010.100313 
Therefore, placing the implant in an ideal prosthetically driven position is of paramount importance in terms of function and long-term implant stability.

In the posterior mandibular region, a lingual undercut is a common finding and can be difficult to manage at times. Recently, Watanabe et al. ${ }^{7}$ categorized the cross-sectional morphology of the mandible. Their data demonstrated that lingual concavity is prevalent in $36 \%$ to $39 \%$ of the study population. Although biomechanically it is best that bucco-lingual implant inclination follows the long axis of the opposing tooth, ignoring the presence of a lingual undercut may lead to perforation of the lingual plate. On the same note, manually fabricated surgical guides following the ideal prosthetic position without considering underlying anatomic limitations may run the risk of lingual plate perforation thus leading to severe surgical complications.

Cone-beam computed tomography (CBCT) has become widely used for implant treatment planning. It provides high resolution and accurate three-dimensional images ${ }^{8}$ and is a vital tool for computer-guided implant surgeries. ${ }^{9}$ In this technique, computer software is used to place the dental implants virtually, followed by transferring the implant position and angulation to the surgical sites by either computergenerated surgical guides or the use of navigation systems. ${ }^{10}$ Clinical trials demonstrated satisfactory outcomes for computer-guided implant treatment plans and a systematic review ${ }^{11}$ found that the estimated deviation measured at entry point and apex was 1.07 and $1.63 \mathrm{~mm}$, respectively.

The incidence of implant placement outside the bony housing in the posterior mandibular region seems to be low because only a case report ${ }^{12}$ was found in the literature. However, a higher perforation rate is suspected because this type of adverse event could have been unnoticed or unreported. There is currently a lack of data concerning implant position in relation to the lingual plate and the significance of the bone morphology, more specifically the lingual concavity, to the incidence of lingual perforation. Therefore, the aims of this computer simulation study are to investigate the incidence of lingual plate perforation in the edentulous mandibular first molar region by placing virtual implants according to the prosthetic outcome, and whether the presence of lingual concavity is related to a higher risk of lingual plate perforation.

\section{MATERIALS AND METHODS}

This study was approved by the institutional review boards of the University of Michigan (09-PAF04299) and was conducted from April to May 2010. The study analyzed anonymized pre-existing images and was exempt from human subjects review.

\section{Image Acquisition and Patient Confidentiality}

All images were acquired with a CBCT machine ${ }^{\ddagger}$ in the Department of Periodontics and Oral Medicine, University of Michigan, School of Dentistry, by board certified oral and maxillofacial radiologists (EB and Sharon L. Brooks, Department of Periodontics and Oral Medicine, School of Dentistry, University of Michigan, Ann Arbor, MI.) between 2005 and 2009. The imaging parameters were set at $120 \mathrm{kVp}$, $18.66 \mathrm{~mA}$, scan time 20 seconds, resolution 0.4 $\mathrm{mm}$, and a field of view that varied based on the region scanned. The scans used in the present study were selected from the CBCT database and were not specifically acquired for this publication.

The CBCT scans of each individual were made anonymously and transferred to a secured file in a password-protected personal desktop computer equipped with an implant planning software pro-

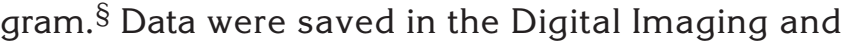
Communications in Medicine format. A list with the codes and corresponding names was created and saved in an encrypted file so that patients' confidentiality was protected and yet retrievable if needed.

\section{Inclusion Criteria}

Images selected for this study had to fulfill the following inclusion criteria: 1) visualization of the posterior mandibular region and opposing maxillary region; 2) patient should have one missing mandibular first molar with the adjacent second premolar present and in a normal position (the imaginary line connecting the cusp tip of canines, central grooves of premolars, and molars was generally smooth); 3 ) the second molar, if present, should be in a normal position as defined previously; 4) maxillary teeth opposing the missing mandibular first molar had to be present to provide information for implant angulation; 5) the edentulous mandibular first molar region had to have sufficient vertical bone height for implant placement as measured from the virtual implant platform to the superior border of inferior alveolar nerve canal (for example, the vertical bone height should be $\geq 12 \mathrm{~mm}$ for the placement of a $10-\mathrm{mm}$-long implant, so that there is a minimum of $2 \mathrm{~mm}$ safety margin between the implant and the inferior alveolar nerve); ${ }^{13} 6$ ) the edentulous mandibular first molar region had to have adequate horizontal bone width for implant placement (for example, a horizontal bone width of $\geq 5$ and $\geq 6 \mathrm{~mm}$ for the placement of 4 and $5 \mathrm{~mm}$ diameter implants, respectively); ${ }^{14}$ and 7 ) the mesiodistal distance of the mandibular first molar edentulous space at the crestal level was $>8 \mathrm{~mm}$ for the placement of one implant of 4 or $5 \mathrm{~mm}$ diameter.

\footnotetext{
$\ddagger \mathrm{i}$-CAT cone-beam computed tomography machine, Imaging Sciences International, Hatfield, PA.

$\S$ InVivoDental, Anatomage, San Jose, CA.
} 
Images were excluded if:1) images were unclear because of scattering or beam-hardening artifacts; 2) bone pathology was present in the posterior mandible; 3 ) the mandibular second premolar was not fully erupted and not in normal position; or 4) the mandibular dimension and interdental space did not fulfill all inclusion criteria.

In this study, the mandibular first molar edentulous region was the site of interest. Based on the inclusion and exclusion criteria, images were selected by two independent examiners (C-YY and H-LC). Disagreements in CBCT scan selection were resolved through discussion.

\section{Virtual Implant Placement and Measurement}

Selected CBCT scans were reconstructed using an implant planning software.| Parallel root-form implants with the following dimensions were selected from an implant database available in the software: $4 \times 10,4 \times 12,5 \times 10$, and $5 \times 12 \mathrm{~mm}$. A single implant was virtually placed into the edentulous mandibular first molar region, as shown in Figure 1.

The same site could receive implants of different dimensions at each measurement, depending on the ridge width and height. Implants were placed based on the following criteria without considering lingual ridge boundaries. Mesiodistally, if the second molar was present, the implant was placed in the middle of the edentulous ridge; otherwise, it was placed $3 \mathrm{~mm}$ away from the root of the adjacent second premolar. ${ }^{15-17}$ Bucco-lingually, the center of the implant platform was positioned along an imaginary line passing through the central fossa of the adjacent teeth (in the presence of the second molar) or the midcrestal ridge (in the absence of the second molar). Apico-coronally, the implant platform was placed at the crestal level. The bucco-lingual angulation of the implant depended on the curvature of the mandibular occlusal plane and the long axis of the opposing maxillary tooth. The implant was placed so that its long axis was in line with that of the opposing tooth. In addition, the functional cusps of the opposing teeth were positioned at the center of the implant. The implant position was verified from different crosssectional and three-dimensional views.

The primary outcome of this study was the distance between the implant tip and the lingual plate (D). Therefore, in the mid-implant cross-sectional view, distance from the surface of the lingual plate to the implant tip was measured with a digital caliper accurate to two decimals. "I Lingual perforation was determined when the implant was out of the plate $(D<0)$ and the incidence was calculated for each implant dimension. Frequencies of implant tip at varying distances (e.g., $<1$ and $2 \mathrm{~mm}$ ) from the lingual plate were counted. Virtual implant placement and measurements were conducted by two calibrated examiners (C-YY and H-LC).

\section{Cross-Sectional Morphology Assessment}

Mandibular cross-sectional morphology $2 \mathrm{~mm}$ coronal to the inferior alveolar nerve was categorized into one of the following three groups (Fig. 2). The crosssectional view $5 \mathrm{~mm}$ distal to the second premolar (in the absence of the second molar) or the middle section of the edentulous span (in the presence of the second molar) was chosen for analysis. A ridge with a narrow base that expands bucco-lingually to a wider crest with a prominent point on the lingual plate, giving rise to a lingual undercut, was classified as an undercut ridge type (type $U$ ). When no obvious lingual undercut was seen, the ridge was categorized into either the convergent ridge type (type C) or the parallel ridge type (type $\mathrm{P})$. The type-C ridge was one where the base of the ridge was wider than its crest. On the other hand, the type-P ridge generally had a more or less parallel ridge form.

\section{Statistical Analyses}

The inter- and intraexaminer agreement was determined by comparing two repeated measurements at three randomly chosen sites taken 1 week apart using Pearson correlation. ${ }^{18}$ The mean distance in millimeters between the implant tip and lingual plate was presented as mean distance \pm SD. The analysis of variance test was used to compare among the three different types of mandibular cross-sectional morphology. The significance level ( $P$ value) was set at 0.05 . All statistical analysis was performed using a statistical package."

\section{RESULTS}

A total of 1,324 subjects were screened and 103 subjects, consisting of 35 males and 68 females, mean age 52.4 years; age range: 23.7 to 76.9 years, were selected. Of the 103 subjects, 118 sites were available for analyses because 15 subjects presented with bilateral absence of the mandibular first molars. Because of the limitation of the mandible dimension, 82 sites were able to receive four different sized implants.

The intraexaminer and interexaminer agreements were 0.93 and 0.89 , respectively. The number and dimensions of implants placed and the incidence of implant tips-lingual plate distance being $<0,1$, or $2 \mathrm{~mm}$ are shown in Table 1 . When $4 \times 10$ or $5 \times 10 \mathrm{~mm}$ implants were selected, no perforation occurred. When $4 \times 12$ or $5 \times 12 \mathrm{~mm}$ implants were chosen, one perforation in a U-type ridge occurred in each group, giving rise to $1.1 \%$ or $1.2 \%$ incidence rate, respectively (Table 1). The lowest mean distances (2.52 to $3.04 \mathrm{~mm}$ ) were found in the undercut (type $U$ ) group but this

\footnotetext{
| InVivoDental, Anatomage.

II InVivoDental, Anatomage.

\# SPSS v.17.0, SPSS, Chicago, IL.
} 

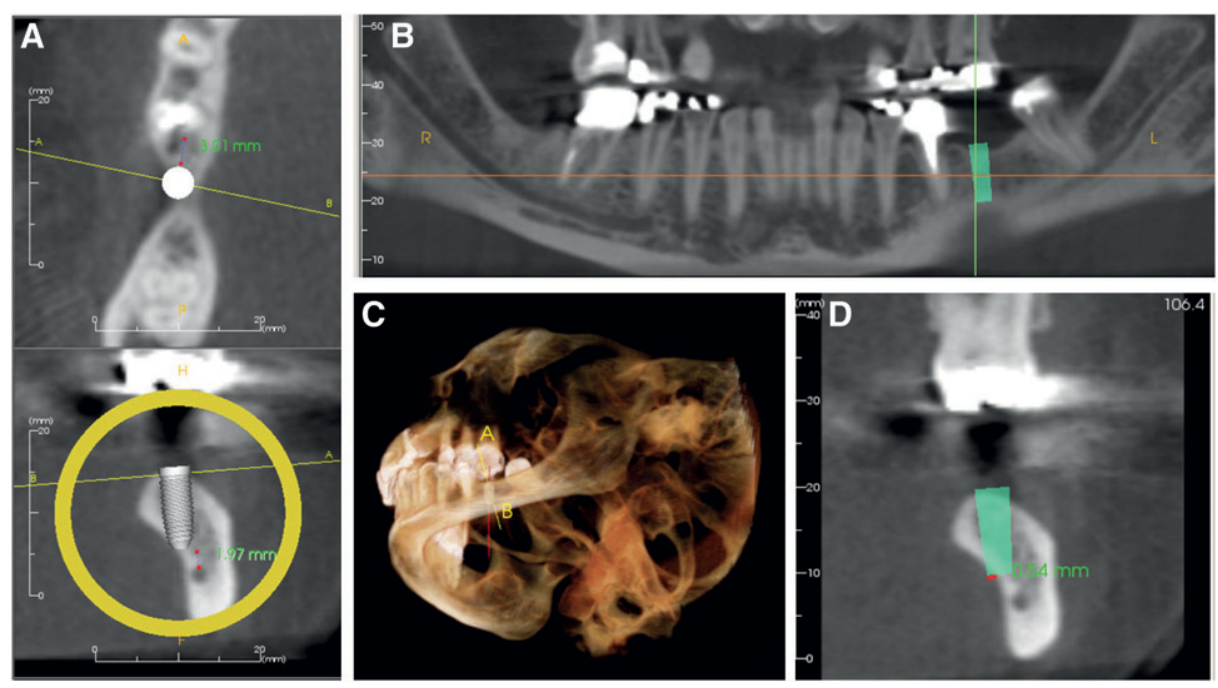

Figure I.

Schematic description of virtual implant placement. A) The implant was 3 and $2 \mathrm{~mm}$ from the mandibular second premolar and the inferior alveolar canal, respectively. B and $\mathbf{C})$ the implant position was verified from reconstructed panoramic and three-dimensional images. D) The distance between the implant tip and the lingual plate was measured with a digital caliper.
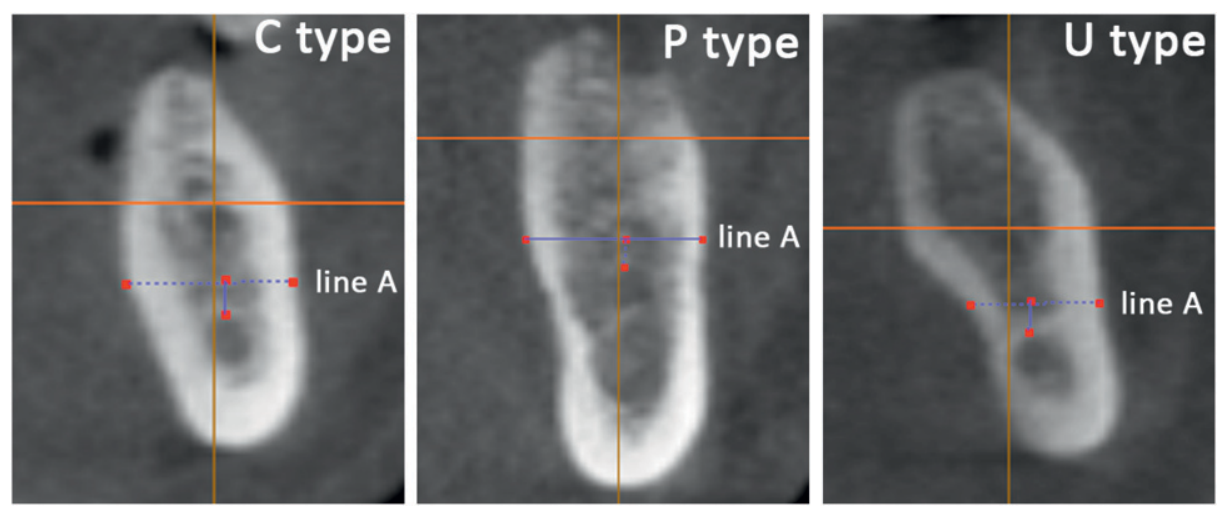

Figure 2.

Three types of cross-sectional posterior mandibular morphology: C, P, and $U$ type. Line A represents a reference line $2 \mathrm{~mm}$ superior to the inferior alveolar nerve.

did not reach statistical significance $(P>0.05)$ (Table 2 ). A total of $2.2 \%$ to $6.1 \%$ of implants, depending on the implant size, were found to be $\leq 1 \mathrm{~mm}$ from the lingual plate and most of them were found in the undercut group (type U) (Table 1).

\section{DISCUSSION}

During osteotomy preparation in the posterior mandibular region, the presence of a lingual concavity calls for special attention because it increases the risk of perforating the lingual plate. ${ }^{19}$ Quirynen et al. ${ }^{20}$ and Tepper et al. ${ }^{21}$ used CT to analyze the morphology and dimensions of the mandible; however, only the interforminal region was examined. To make it easier to identify ridge shape and recognize potential perfora- tion risks, we classified the ridge to three types: $U$ (undercut), C (convergent), and $\mathrm{P}$ (parallel), which had a prevalence rate of $13.6 \%, 20.4 \%$, and $66 \%$, respectively, in this study. Similarly, Watanabe et al. ${ }^{22}$ classified posterior mandibular cross-sectional morphology into types $\mathrm{A}, \mathrm{B}$, and $\mathrm{C}$. According to the outlines of the lingual and buccal plates, types $\mathrm{A}, \mathrm{B}$, and $\mathrm{C}$, were described as round on the buccal side and concave on the lingual side, concave on the buccal side and round on the lingual side, and roundshaped on both sides respectively. Their results showed that type C (round) was the most common (59\% to $61 \%$ ), followed by type A (lingual concavity) (36\% to $39 \%$ ). Another study 23 found $80 \%$ of their study population had $\geq 2 \mathrm{~mm}$ lingual concavity measured at the deepest portion of submandibular fossa. Therefore, it is certain that varying degrees of lingual concavities are present in a significant number of populations.

Lingual plate perforation is difficult to assess from radiographic images because of potential artifacts around implants. ${ }^{24}$ The beam-hardening effect of implants in CT or CBCT images complicates the establishment of a definitive diagnosis of lingual perforation, hindering investigations on the incidence of lingual perforations after implant placement. The beam-hardening effect is an inherent artifact resulting from the polychromatic absorption of low-energy $\mathrm{x}$-ray photons by metallic objects resulting in an exiting $\mathrm{x}$-ray beam that contains mainly high-energy x-ray photons (e.g., a harder beam). ${ }^{25}$ Although artifact reduction technique algorithms have been developed, they are computationally demanding and time consuming. ${ }^{25}$ Unless potential artifact caused by metallic objects (e.g., dental implants) can be resolved, the use of CT/CBCT for postoperative evaluation is not justifiable at this time.

This simulation study was designed to estimate the incidence of lingual plate perforation and assess 
Table I.

\section{Frequency Distribution of Implant Position Relative to Lingual Plate With Different Implant Size and Threshold Among Three Morphology Types}

\begin{tabular}{lcrrrrr}
\hline $\begin{array}{l}\text { Implant } \\
\text { Size/Threshold }\end{array}$ & & & & & & \\
\hline $4 \times 10 \mathrm{~mm}$ & $\mathrm{n}$ & 16 & 24 & 78 & 118 & \\
& $\leq 2 \mathrm{~mm}$ & 0 & 1 & 18 & 19 & 16.0 \\
& $\leq 1 \mathrm{~mm}$ & 0 & 0 & 5 & 5 & 4.2 \\
& $<0 \mathrm{~mm}$ & 0 & 0 & 0 & 0 & 0.0 \\
$4 \times 12 \mathrm{~mm}$ & $\mathrm{n}$ & 9 & 20 & 58 & 87 & \\
& $\leq 2 \mathrm{~mm}$ & 0 & 3 & 16 & 19 & 21.8 \\
& $\leq 1 \mathrm{~mm}$ & 0 & 0 & 2 & 2 & 2.2 \\
& $<0 \mathrm{~mm}$ & 0 & 0 & 1 & 1 & 1.1 \\
$5 \times 10 \mathrm{~mm}$ & $\mathrm{n}$ & 15 & 24 & 71 & 110 & \\
& $\leq 2 \mathrm{~mm}$ & 0 & 3 & 18 & 21 & 19.1 \\
& $\leq 1 \mathrm{~mm}$ & 0 & 0 & 5 & 5 & 4.5 \\
& $<0 \mathrm{~mm}$ & 0 & 0 & 0 & 0 & 0.0 \\
$5 \times 12 \mathrm{~mm}$ & $\mathrm{n}$ & 8 & 20 & 54 & 82 & \\
& $\leq 2 \mathrm{~mm}$ & 0 & 4 & 17 & 21 & 25.6 \\
& $\leq 1 \mathrm{~mm}$ & 0 & 1 & 4 & 5 & 6.1 \\
& $<0 \mathrm{~mm}$ & 0 & 0 & 1 & 1 & 1.2 \\
\hline
\end{tabular}

Table 2.

\section{Mean Distance Between Implant Tip and Lingual Plate Among Three Morphology Types}

\begin{tabular}{lccc}
\hline \multirow{2}{*}{ Implant Dimensions $(\mathrm{mm})$} & \multicolumn{3}{c}{ Mean Distance in Millimeters (SD) } \\
\cline { 2 - 4 } & C Type & P Type & $\cup$ Type \\
\hline $4 \times 10$ & $3.63(0.78)$ & $3.31(0.97)$ & $3.04(1.36)$ \\
$4 \times 12$ & $3.39(0.80)$ & $3.23(1.04)$ & $2.70(1.24)$ \\
$5 \times 10$ & $3.41(0.80)$ & $2.98(0.95)$ & $2.82(1.20)$ \\
$5 \times 12$ & $3.25(0.96)$ & $2.92(1.03)$ & $2.52(1.20)$ \\
\hline
\end{tabular}

whether the presence of a lingual concavity increases this risk. The validity of this study design relies on the accuracy of CBCT images. ${ }^{26-30}$ The elimination of implant-related beam-hardening artifacts through the virtual implant placement is also advantageous. One challenge encountered in this study was the wide range of possible implant positions in each simulated scenario. Therefore, suggestions provided in the literature $^{4,15-17,31}$ were formulated into our implant placement guidelines. From a biomechanical standpoint, the direction of occlusal loading determines the bucco-lingual inclination of the implant. ${ }^{4}$ The authors placed the implant virtually following the long axis of the opposing tooth, thus obeying biomechanical principles. In addition, the intra- and interexaminer agreement was high, suggesting that the implant placement was reproducible.

The predicted incidence of lingual plate perforation was $1.1 \%$ to $1.2 \%$, depending on the diameter of the implant. If implant placement errors are considered, ${ }^{11}$ a distance of $\leq 1 \mathrm{~mm}$ between the virtual implant and the lingual plate in this computer simulated scenario could be extrapolated to imply a lingual plate perforation in a clinical situation. The frequency of implants being $\leq 1 \mathrm{~mm}$ to the lingual plate was found to be $2.2 \%$ to $6.1 \%$, with most of the cases occurring in the undercut group (type- $U$ ridge). Compared to the other groups, the mean distance between the implant tip and the lingual plate is not significantly smaller in the undercut group (type-U ridge). However, most virtual implants that were in close proximity to the lingual plate $(\leq 1 \mathrm{~mm})$ were in the type- $U$ ridge. This finding suggests that a lingual concavity is a predisposing factor for lingual perforation. Interestingly, even though a small number of subjects with lingual concavity had the virtual implant close to or perforating the lingual plate, a considerably larger number did not. Hence, other factors, such as the occlusal plane in relation to the mandible inclination, may determine the relative risk of a lingual concavity in predisposing to a lingual plate perforation.

At the time of implant treatment planning in the posterior mandible, it is advisable to palpate the lingual side of the ridge to detect the presence of a lingual concavity. When a significant lingual concavity is encountered, a CT or CBCT scan with a radiographic guide may be indicated preoperatively so that the implant angulation in relation to this anatomic limitation can be assessed. Tapered implants may be used instead of parallel ones. Alternatively, an implant might be placed off-axially to avoid the concavity and restored with an angled abutment if there is a concern of lingual plate perforation after axial placement.

\section{CONCLUSIONS}

This study demonstrates a novel experimental design by which the spatial relation between the implant and the lingual plate in the mandibular first molar region was investigated. Results indicated $1.1 \%$ to $1.2 \%$ 
incidence of lingual plate perforation, which occurred in the undercut (type $U$ ) ridge, when parallel implants with 4- or 5- $\mathrm{mm}$ wide and 10- or 12- mm long implants were placed in edentulous ridges with $\geq 5$ or $6 \mathrm{~mm}$ in width (for 4 - or 5- mm wide implants) and $\geq 12$ or $14 \mathrm{~mm}$ in height measured from implant platform to the inferior alveolar nerve canal (for 10- or 12- mm long implants). The result obtained from this study question the routine use of CBCT for implant therapy especially after the implant was inserted. Future research should aim at identifying factors that predispose subjects with lingual concavity to a higher risk of lingual plate perforation.

\section{ACKNOWLEDGMENTS}

The authors thank Dr. Sharon L. Brooks, professor, Department of Periodontics and Oral Medicine, School of Dentistry, University of Michigan, for her expertise and Chia-Ning Wang, PhD candidate, Department of Biostatistics, School of Public Health, University of Michigan, for statistical analysis consultation. This paper was partially supported by the University of Michigan Periodontal Graduate Student Research Fund. The authors report no conflicts of interest related to this study.

\section{REFERENCES}

1. Adell R, Lekholm U, Rockler B, Brånemark PI. A 15 -year study of osseointegrated implants in the treatment of the edentulous jaw. Int J Oral Surg 1981;10: 387-416.

2. Scheller H, Urgell JP, Kultje C, et al. A 5-year multicenter study on implant-supported single crown restorations. Int J Oral Maxillofac Implants 1998;13: 212-218.

3. Becker CM, Kaiser DA. Surgical guide for dental implant placement. J Prosthet Dent 2000;83:248-251.

4. Misch CE, Bidez MW. Implant-protected occlusion: A biomechanical rationale. Compendium 1994;15:1330, 1332, 1334 passim; quiz 1344.

5. Reilly DT, Burstein AH. The elastic and ultimate properties of compact bone tissue. J Biomech 1975; 8:393-405.

6. Lin CL, Wang JC, Ramp LC, Liu PR. Biomechanical response of implant systems placed in the maxillary posterior region under various conditions of angulation, bone density, and loading. Int J Oral Maxillofac Implants 2008;23:57-64.

7. Watanabe H, Mohammad Abdul M, Kurabayashi T, Aoki H. Mandible size and morphology determined with CT on a premise of dental implant operation. Surg Radiol Anat 2010;32:343-349.

8. White SC. Cone-beam imaging in dentistry. Health Phys 2008;95:628-637.

9. Van Assche N, van Steenberghe D, Guerrero ME, et al. Accuracy of implant placement based on pre-surgical planning of three-dimensional cone-beam images: A pilot study. J Clin Periodontol 2007;34:816-821.

10. Ganz SD. Computer-aided design/computer-aided manufacturing applications using $\mathrm{CT}$ and cone beam CT scanning technology. Dent Clin North Am 2008;52: 777-808, vii.
11. Schneider D, Marquardt P, Zwahlen M, Jung RE. A systematic review on the accuracy and the clinical outcome of computer-guided template-based implant dentistry. Clin Oral Implants Res 2009;20(Suppl. 4): 73-86.

12. Berberi A, Le Breton G, Mani J, Woimant H, Nasseh I. Lingual paresthesia following surgical placement of implants: Report of a case. Int $J$ Oral Maxillofac Implants 1993;8:580-582.

13. Sammartino G, Marenzi G, Citarella R, Ciccarelli R, Wang HL. Analysis of the occlusal stress transmitted to the inferior alveolar nerve by an osseointegrated threaded fixture. J Periodontol 2008;79: 1735-1744.

14. Chiapasco M, Abati S, Romeo E, Vogel G. Clinical outcome of autogenous bone blocks or guided bone regeneration with e-PTFE membranes for the reconstruction of narrow edentulous ridges. Clin Oral Implants Res 1999;10:278-288.

15. Tarnow DP, Cho SC, Wallace SS. The effect of interimplant distance on the height of inter-implant bone crest. J Periodontol 2000;71:546-549.

16. Gastaldo JF, Cury PR, Sendyk WR. Effect of the vertical and horizontal distances between adjacent implants and between a tooth and an implant on the incidence of interproximal papilla. J Periodontol 2004; 75:1242-1246.

17. Romeo E, Lops D, Rossi A, Storelli S, Rozza R, Chiapasco M. Surgical and prosthetic management of interproximal region with single-implant restorations: 1-year prospective study. J Periodontol 2008; 79:1048-1055.

18. Pearson K. Determination of the coefficient of correlation. Science 1909;30:23-25.

19. Greenstein G, Cavallaro J, Tarnow D. Practical application of anatomy for the dental implant surgeon. $J$ Periodontol 2008;79:1833-1846.

20. Quirynen M, Mraiwa N, van Steenberghe D, Jacobs R. Morphology and dimensions of the mandibular jaw bone in the interforaminal region in patients requiring implants in the distal areas. Clin Oral Implants Res 2003; 14:280-285.

21. Tepper G, Hofschneider UB, Gahleitner A, Ulm C. Computed tomographic diagnosis and localization of bone canals in the mandibular interforaminal region for prevention of bleeding complications during implant surgery. Int J Oral Maxillofac Implants 2001;16: 68-72.

22. Watanabe H, Mohammad Abdul M, Kurabayashi T, Aoki H. Mandible size and morphology determined with CT on a premise of dental implant operation. Surg Radiol Anat 2010;32(4):343-349.

23. Parnia F, Fard EM, Mahboub F, Hafezeqoran A, Gavgani FE. Tomographic volume evaluation of submandibular fossa in patients requiring dental implants. Oral Surg Oral Med Oral Pathol Oral Radiol Endod 2010;109:e32-e36.

24. Draenert FG, Coppenrath E, Herzog P, Müller S, Mueller-Lisse UG. Beam hardening artefacts occur in dental implant scans with the NewTom cone beam CT but not with the dental 4-row multidetector CT. Dentomaxillofac Radiol 2007;36:198-203.

25. Scarfe WC, Farman AG. What is cone-beam CT and how does it work? Dent Clin North Am 2008;52:707730 .

26. Lascala CA, Panella J, Marques MM. Analysis of the accuracy of linear measurements obtained by cone 
beam computed tomography (CBCT-NewTom). Dentomaxillofac Radiol 2004;33:291-294.

27. Ito K, Gomi Y, Sato S, Arai Y, Shinoda K. Clinical application of a new compact CT system to assess 3-D images for the preoperative treatment planning of implants in the posterior mandible: A case report. Clin Oral Implants Res 2001;12:539-542.

28. Pinsky HM, Dyda S, Pinsky RW, Misch KA, Sarment DP. Accuracy of three-dimensional measurements using cone-beam CT. Dentomaxillofac Radiol 2006; 35:410-416.

29. Kobayashi K, Shimoda S, Nakagawa Y, Yamamoto A. Accuracy in measurement of distance using limited cone-beam computerized tomography. Int J Oral Maxillofac Implants 2004;19:228-231.
30. Araki K, Maki K, Seki K, et al. Characteristics of a newly developed dentomaxillofacial X-ray cone beam CT scanner (CB MercuRay): System configuration and physical properties. Dentomaxillofac Radiol 2004;33:51-59.

31. Buser D, Martin W, Belser UC. Optimizing esthetics for implant restorations in the anterior maxilla: Anatomic and surgical considerations. Int $J$ Oral Maxillofac Implants 2004;19(Suppl.):43-61.

Correspondence: Professor Hom-Lay Wang, 1011 North University Ave., Ann Arbor, MI 48109-1078. Fax: 734/ 936-0374; e-mail: homlay@umich.edu.

Submitted May 24, 2010; accepted for publication June 28, 2010. 